\title{
IdeAs
}

Idées d'Amériques

16 | 2020

Les marges créatrices : intellectuel.le.s afrodescendant.e.s et indigènes auX Amériques, XIX-XXe siècle

\section{Ballot Issues : la démocratie directe et la société américaine dans les urnes}

Donna Kesselman

\section{OpenEdition \\ Journals}

Édition électronique

URL : http://journals.openedition.org/ideas/9841

DOI : 10.4000/ideas.9841

ISSN : 1950-5701

Éditeur

Institut des Amériques

\section{Référence électronique}

Donna Kesselman, «Ballot Issues : la démocratie directe et la société américaine dans les urnes », IdeAs [En ligne], 16 | 2020, mis en ligne le 01 octobre 2020, consulté le 19 octobre 2020. URL : http:// journals.openedition.org/ideas/9841; DOI : https://doi.org/10.4000/ideas.9841

Ce document a été généré automatiquement le 19 octobre 2020

\section{c) (i)}

IdeAs - Idées d'Amériques est mis à disposition selon les termes de la licence Creative Commons Attribution - Pas d'Utilisation Commerciale - Pas de Modification 4.0 International. 


\title{
Ballot Issues : la démocratie directe et la société américaine dans les
}

\section{urnes}

\author{
Donna Kesselman
}

1 Le jour où ils vont élire leurs représentants, les citoyens des deux tiers des États américains participent à la démocratie directe car ils sont aussi appelés à se prononcer sur de la législation ou amender la constitution locale sans le consentement de ces mêmes élus. Souhaitant contourner les machines politiques corrompues, soucieux de l'influence politique dont jouissaient les magnats de l'industrie sur le processus démocratique, les réformateurs et éducateurs de l'ère progressiste au début $\mathrm{du} \mathrm{xx}^{\mathrm{e}}$ siècle ont privilégié les vertus de la volonté populaire. La collecte du nombre prescrit de signatures d'électeurs mettant sur le bulletin de vote une question d'intérêt public (Ballot Issue) donnerait la parole et un contre-pouvoir aux citoyens. Un siècle plus tard, l'activisme plébiscitaire connaît un regain de popularité : 121 referendums auront été soumis au vote dans 34 États aux États-Unis pour l'Election Day, le 3 novembre 2020.

2 La démocratie directe fait intervenir une expression populaire dans la politique publique, la gouvernance et la mise à l'agenda des préoccupations citoyennes. Ce faisant, elle s'avère un point d'observation privilégié de la société américaine à partir des cultures politiques locales, ces champs de batailles autour d'enjeux sociétaux comme le droit à l'avortement ou la consommation de la marijuana, et des questions brûlantes d'actualité, tel le statut des chauffeurs VTC en Californie en 2020. Elle offre un aperçu plus nuancé du consensus politique et de son évolution que ne laisse apparaitre le portrait d'un pays polarisé entre États « rouges » conservateurs qui votent républicain ou États «bleus » progressistes qui votent démocrate lors des élections présidentielles.

3 Parmi les différents mécanismes référendaires - «Ballot Issues » ou «Propositions » sont des termes génériques - ils sont 24 États plus Washington D.C., à autoriser la ballot initiative, le référendum d'initiative populaire, le plus fréquent, l'outil offensif où les pétitionnaires soumettent au plébiscite la proposition d'une loi ou d'un amendement à 
la constitution de l'État ${ }^{1}, 33$ à autoriser l'outil défensif du veto referendum ou referendum tout court, qui permet à l'électorat d'abroger une loi (ou une partie d'une loi). Ces États se trouvent surtout dans l'Ouest, mais $70 \%$ de la population peut être appelée à légiférer lorsque l'on ajoute la démocratie directe municipale. La période de circulation pendant laquelle les pétitionnaires sont autorisés à obtenir les signatures nécessaires pour mettre la proposition au scrutin varie de 60 jours au Massachussetts à quatre ans en Floride.

Le dernier des trois mécanismes de la démocratie directe est le référendum révocatoire (recall), procédure, autorisée dans 39 États, par laquelle les électeurs peuvent démettre de ses fonctions un élu. En 2003, les électeurs de Californie ont révoqué l'ancien gouverneur démocrate Gray Davis pour le remplacer par l'acteur-homme d'affaires Arnold Schwarzenegger. En 2012, le gouverneur républicain du Wisconsin, Scott Walker, en même temps que huit élus, ont fait l'objet d'un référendum révocatoire en protestation contre la politique de remise en cause des droits syndicaux pour les travailleurs du secteur public. Lancée par le Parti démocrate local et des syndicats nationaux dans le but de canaliser le véritable soulèvement populaire qui a culminé par l'occupation du congrès de l'État, l'élection a apporté quelques sièges aux démocrates sans pour autant réussir à évincer le gouverneur Walker. En 2018, des 123 élus qui ont fait face à un referendum à l'échelle locale, 77 (63,6 \%) ont été révoqués ${ }^{2}$.

\section{La démocratie directe dans les États américains : les tendances récentes ${ }^{3}$}

5 Champ de bataille des guerres culturelles, la démocratie directe s'avère un indicateur des avancées des camps en présence. A la suite des reculs dans l'accès au droit de l'avortement obtenus par le camp "pro-life » depuis les années 1990, un consensus semblait se dégager au sein de chaque contexte local et contre les mesures les plus restrictives. L'obligation pour le médecin pratiquant un avortement sur mineure d'en informer le parent (ou tuteur) a été repoussée en Californie (2008) mais approuvée dans l'Alaska (2010) et le Montana (2012). Les électeurs ont refusé l'introduction d'une définition radicale de la vie humaine à partir du moment de la fécondation qui aurait donné lieu à l'interdiction de tout avortement (Colorado 2008, 2010 et 2014, Mississippi 2011, Dakota du Nord 2014) et l'interdiction de l'avortement sauf dans les cas extrêmes (viol, inceste, risque de vie de la mère) dans le Dakota du Sud (2008). Par un retour de balancier, le Colorado sera le théâtre d'un référendum en novembre 2020 qui vise à interdire l'avortement à partir de la $22^{\mathrm{e}}$ semaine de la grossesse ${ }^{4}$, et des initiatives restrictives seront soumises au vote dans plusieurs États en 2022. La croisade des opposants à l'avortement semble entrer dans une nouvelle ère, celle de l'après Roe $v$. Wade. Dans la perspective où la Cour suprême reviendrait sur sa décision historique, l'Alabama et la Virginie occidentale ont voté un amendement (2018) qui empêcherait toute mesure future autorisant l'avortement dans leur État $^{5}$; une proposition similaire est soumise au scrutin en 2020 en Louisiane.

Dans une dynamique inverse, le Nevada sera en novembre le premier État à soumettre à la population l'abrogation de l'amendement qui définit le mariage comme étant entre un homme et une femme pour introduire la notion de mariage d'un couple sans considération de genre (Mariage Regardless of Gender Amendment). Des amendements d'interdiction avaient été votés dans de nombreux États avant la décision de la Cour 
suprême qui a légalisé le mariage entre personnes de même sexe sur tout le territoire (Obergefell v. Hodges, 2015).

7 Dans l'esprit des progressistes d'antan, la population se saisit des outils plébiscitaires pour participer au processus d'équilibre des pouvoirs. En plus des 15 États où elle a déjà été mise en place pour les élus du congrès local (36 pour des gouverneurs), la limitation de la durée des mandats (term limits) est soumise en 2020 aux électeurs de l'Arizona. Comme réponse populaire au gerrymandering, le découpage de circonscriptions électorales par les élus dans le but de favoriser l'élection d'un parti politique, ceux de l'Arkansas, du Dakota du Nord, du Missouri, de l'Oregon, et de Virginie sont sollicités en 2020 pour rejoindre les États qui disposent de commissions indépendantes chargées d'établir des circonscriptions électorales selon des critères précis et plus équitables ${ }^{6}$.

La démocratie directe est le terrain où s'organise l'opposition à l'arrêt de la Cour suprême Citizens United v. Federal Election Commission (2010) qui a interdit à l'État fédéral de restreindre les dépenses de communication politique par des organisations (entreprises, syndicats, etc.) qui ne sont pas faites en coopération avec un candidat (independent expenditures). En reconnaissant leur droit à la «liberté d'expression »du premier amendement de la Constitution des États-Unis, la Cour a assimilé ces dernières à des individus. L'augmentation fulgurante des dépenses électorales qui s'en est suivie doublant entre les élections présidentielles de 2008 et 2016, en hausse encore en 2020 aurait renforcé l'influence des plus fortunés, notamment les entreprises. Suivant les électeurs dans les États de Californie et de Washington (2016) qui se sont prononcés pour réserver les droits et libertés constitutionnels aux êtres humains par voie d'un amendement à la Constitution américaine - la seule riposte possible à une décision de la Cour suprême - et ceux du Massachussetts (2018) sous la devise «People Govern, Not Money ", les électeurs de l'Alaska et de l'Oregon se prononceront sur cette perspective le 3 novembre.

\section{La démocratie directe est-elle démocratique?}

9 Les détracteurs de la démocratie directe reprennent à leur compte les critiques classiques du processus référendaire aux dépens de la démocratie représentative ${ }^{7}$. Puisqu'il opère une mobilisation à grande échelle et demande d'approuver ou de rejeter un texte en bloc, le référendum serait incompatible avec les attendus de la délibération démocratique, dont la recherche de compromis, ce qui aggraverait les conflits et nuirait aux intérêts des minorités. Quant à la prise de décisions, le peuple est-il compétent pour légiférer? Les électeurs californiens de la Proposition 13 en 1978 qui ont voté pour limiter les impôts fonciers que l'État peut prélever avaient-ils conscience des réductions draconiennes des dépenses publiques qui allaient s'ensuivre? La Proposition 15 en 2020 projette de rétablir l'imposition foncière en Californie, sur les entreprises à haute valeur immobilière, au nom du financement des écoles et des services publics.

Une autre critique du référendum relève de la modalité de formulation de la question, à savoir l'avantage procuré par celui qui la pose. Un exemple aux États-Unis concerne les initiatives dites des «droits civiques». Évoquant le langage d'égalité de traitement, s'adressant à des citoyens particulièrement sensibles à l'accès aux droits comme les minorités, elles introduisent un amendement à la constitution d'un État qui affirme typiquement : «The state shall not discriminate against, or grant preferential treatment to any 
individual or group on the basis of race, sex, color, ethnicity, or national origin in the operation of public employment, public education, or public contracting ». Or, la doctrine de neutralité des institutions en matière de race (colorblind institutions) a été véhiculée historiquement aux États-Unis pour masquer la discrimination de fait. Ainsi, les premières Civil Rights Initiatives approuvées en Californie (1996) et Washington (1998) ont donné lieu à l'élimination des politiques de discrimination positive à l'université, aboutissant à une réduction de la population estudiantine issue des minorités dans des filières sélectives telles que le droit et la médecine. La Proposition 16 en Californie en 2020 amenderait cette interdiction par une révision constitutionnelle.

11 La démocratie est mise à mal en matière de financement des campagnes autour des referendums : l'argent coule à flots dans la publicité, surtout depuis l'arrêt Citizens United. Si l'image romantique de militants bénévoles dévoués, en quête de signataires, s'applique encore à certaines initiatives citoyennes, elle s'efface devant l'industrie juteuse de collecte de signatures et de communication politique. Plus de 400 millions de dollars seront dépensés en 2020.

12 Ainsi, le référendum le plus en vue en 2020 est celui lancé en Californie par des magnats du capitalisme de plateforme, la société de transport par chauffeur de VTC Uber, rejointe par son concurrent Lyft et les sociétés de livraison de plats cuisinés DoorDash, et de livraison de produits alimentaires Instacart et Postmates, qui consacrent 110 millions de dollars (92,6 millions d'euros) à ce qui est l'une des campagnes référendaires les plus coûteuses de l'histoire étasunienne. L'objectif est d'exempter ces entreprises de la loi $\mathrm{AB} 5$, promulguée le $1^{\mathrm{er}}$ janvier 2020, qui introduit la présomption de salariat pour les travailleurs. Or, ainsi que l'ont expliqué ces deux leaders du marché VTC dans leur dossier d'entrée en bourse en 2019, l'embauche de salariés, avec les coûts salariaux et fiscaux que cela représente, est contradictoire avec leur modèle économique. La Proposition 22 définit les chauffeurs et livreurs travaillant par l'intermédiaire des plateformes comme des travailleurs indépendants et met en place une politique en matière de rémunération et de conditions de travail «spécifiques aux chauffeurs et aux entreprises de plateforme ". Au-delà des milieux d'affaires et des chambres de commerce, des associations d'Hispaniques et le NAACP (National Association for the Advancement of Colored People) de Californie ont apporté leur soutien à la Proposition 22 depuis sa promotion en termes de création d'emplois pour les travailleurs à bas revenus et qui procure une grande flexibilité dans les horaires.

Or, cette formulation alléchante de la question référendaire sert à escamoter, selon ses détracteurs, les avantages supérieurs qu'occasionnerait une requalification salariale pour les chauffeurs ainsi que les enjeux pour l'avenir du salariat aux États-Unis de ce qui reviendrait à introduire, pour la première fois dans ce pays, une troisième figure du marché du travail. Quel que soit le résultat, la Proposition 22 illustre un autre travers de la démocratie directe, les coûts d'opportunité pour les adversaires d'une proposition. Comme pour toutes les initiatives anti-ouvrières, les syndicats doivent consacrer des ressources financières précieuses à, dans le meilleur des cas, une victoire à la Pyrrhus. La Proposition 22 augure mal car les 1,8 millions de dollars qu'y consacrent les syndicats californiens font pâle figure devant le trésor de guerre des compagnies et de surcroît, ces entreprises ont directement accès à l'électorat californien, via les adresses email et les numéros de téléphone de leurs clients. 


\section{Conclusion}

14 Ainsi, la boucle est bouclée. Un siècle plus tard, les entreprises et les groupes d'intérêt à fort pouvoir économique se retrouvent de nouveau en butte à des critiques pour l'influence disproportionnée qu'ils exercent sur le processus électoral mais cette fois, sur le terrain de la démocratie directe lui-même. La capacité de monnayer leur modèle économique sous la forme édulcorée d'un référendum pourrait permettre aux sociétés de plateforme de contourner la loi californienne. En même temps, dans d'autres cas, plutôt que d'affaiblir la démocratie représentative, le plébiscite populaire lui sert de correctif et d'outil démocratique par excellence. Pour les observateurs de la société américaine, le prisme de la démocratie directe propose un formidable aperçu des microcosmes de cultures politiques et sociétales locales, car chaque expérience référendaire est localisée et contextualisée.

\section{NOTES}

1. Les États qui autorisent le référendum d'initiative populaire sont: Alaska, Arizona, Arkansas, Californie, Colorado, Floride, Idaho, Illinois, Massachusetts, Michigan, Mississippi, Missouri, Montana, Nebraska, Nevada, Dakota du Nord, Ohio, Oklahoma, Oregon, Dakota du Sud, Utah, Washington, Wyoming, District de Columbia.

2. Donna Kesselman, «Direct Democracy on Election Day: Ballot Measures as Measures of American Democracy », TransAtlantica, 2011/1, https://journals.openedition.org/transatlantica/ 5279

3. Pour l'actualité des questions référendaires voir: Ballotpedia, http://ballotpedia.org/, National Conference of State Legislatures, "Statewide Ballot Measures Database », Initiative \& Referendum Institute, University of Southern California, www.iandrinstitute.org.

4. Selon l'arrêt Roe $v$. Wade du 22 janvier 1973 l'avortement est un droit constitutionnel pour toutes les Américaines pendant le premier trimestre, mais qui s'efface au profit de la possibilité de régulation par les États dès la constatation de la viabilité du fotus, soit entre la $24^{\mathrm{e}}$ et $28^{\mathrm{e}}$ semaine (sauf si la santé ou la vie de la mère sont en danger), mais dont le délai est désormais contesté au nom du progrès de la science.

5. L'amendement dans l'Alabama : " [would] make it state policy to "recognize and support the sanctity of unborn life and the rights of unborn children, including the right to life" and to state that no provisions of the constitution provide a right to an abortion or require funding of abortions ".

6. Daniel A. Smith, Caroline J. Tolbert, Educated by Initiative: The Effects of Direct Democracy on Citizens and Political Organizations in the American States, Ann Arbor, The University of Michigan Press, 2007.

7. Laurence Morel, «Référendum et volonté populaire: la critique démocratique du référendum », Participations, 2018/1, Nº 20, p. 53-84. 


\section{AUTEUR}

\section{DONNA KESSELMAN}

Donna Kesselman est professeure à l'Université Paris-Est Créteil (IMAGER EA 3958). Elle publie largement sur le travail, l'emploi et les relations professionnelles des États-Unis, dans une perspective comparative entre les Amériques et l'Europe. Elle a récemment coédité la Chronique internationale de l'IRES sur les chauffeurs VTC dans sept pays, elle a récemment publié des articles sur les élections américaines dans les revues Pouvoirs $\left(n^{\circ} 172\right)$ et IdeAs $\left(n^{\circ} 16\right)$, elle a été porteuse de l'ANR "Les zones grise d'emploi et du travail". 